\title{
EFFICIENT AND ROBUST CREATION OF STRUCTURAL COMPONENT MODELS FOR THERMO-ELASTIC ANALYSIS OF MACHINE TOOLS
}

\author{
S. Schroeder*, S. Ihlenfeldt, L. Penter \\ Technische Universitaet Dresden, Chair of Machine Tools Development and Adaptive Controls, Dresden, Germany \\ ${ }^{*}$ Corresponding author; e-mail: steffen.schroeder1@tu-dresden.de
}

\begin{abstract}
To control the thermal behavior of machine tools, numerous measures are used today. With the help of thermal machine models, well-founded predictions can be made for the selection and design of these measures. For creation and simulation of thermo-elastic models of structural components and a high effort is still necessary. In the paper, a procedure is presented to reduce this effort. A combination of existing methods and new approaches is used. This includes methods of mathematical model order reduction to reduce computing costs and robust mesh algorithms that process even slightly defective geometries.
\end{abstract}

\section{Keywords:}

Thermal behavior; machine tool; thermal model; meshing

\section{INTRODUCTION}

The demand for higher accuracy is a constant challenge for metal-cutting machine tools. According to estimates of machine manufacturers, the largest share of machining errors is due to thermo-elastic behavior [Putz 2018]. The improvement of this behavior is therefore of particular relevance.

There are numerous known measures to control the thermo-elastic behavior. There are compensatory measures. These influence the thermal processes through design measures with the aim of minimizing the displacement of the TCP (Tool Center Point). Examples are the shape optimization of structural components [Mori 2009], the reduction of the heat input of friction-based and electrical heat sources, the temperature control of internal heat sources via cooling circuits [Donmez 2007] and air conditioning of the machine environment [Putz 2018]. Alternatively, control-based correction measures are used to predict the remaining TCP displacements by models and to correct them by inverse movements of the feed axes [Mayr 2018].

A well-founded evaluation, selection and design of these measures requires a broad knowledge base. This this is obtained by analyses of the thermo-elastic machine behavior. The analyses must determine the cause-andeffect relationships of the thermo-elastic behavior so that effective actions can be taken at the causal phenomenon.

This paper presents a comprehensive and efficient modeling and simulation methodology especially for the thermal analysis of machine tools. A modeling concept for
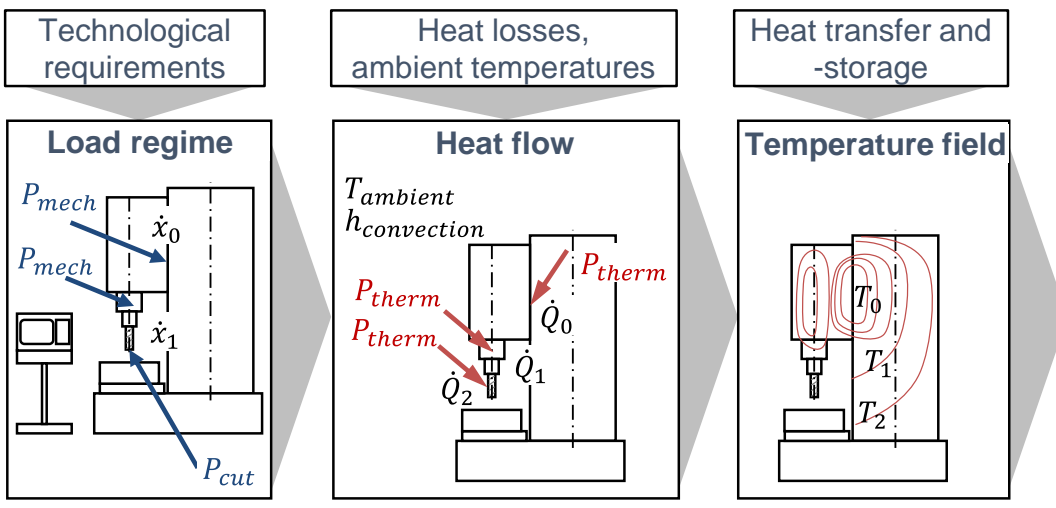
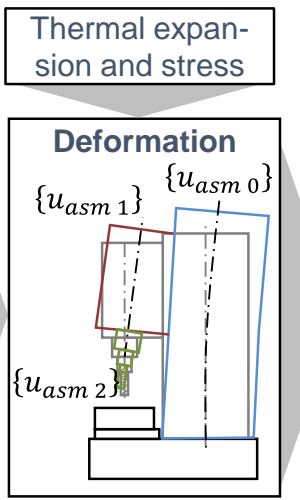

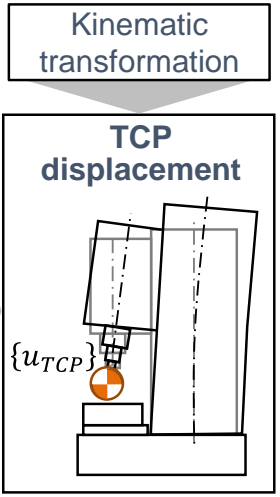

Fig. 1: Accuracy relevant behavior of the thermo-elastic chain of effects (according to [Kauschinger 2016]) MM Science Journal | 2021 | Special Issue on ICTIMT2021 
machines based on a construction kit of model elements is introduced, where the elements represent essential behavioral components. Subsequently, the creation of model elements for the detailed representation of structural solid-state parts is discussed.

A novelty is the application of state-of-the-art model order reduction (MOR) and meshing techniques in regard to thermal analysis. Furthermore, the workflow is implemented using a modular chaining of tools. This allows an easy transfer of the methodology into established tool chains of the industry.

\section{ANALYSES OF THERMO-ELASTIC BEHAVIOR}

\subsection{Thermo-elastic behavior}

The thermo-elastic behavior of machine tools is characterized by processes that extend over several physical areas. These processes form the thermo-elastic functional chain and are shown in Fig. 1.

The machines are composed of axle assemblies that move relative to each other to generate a relative movement and a process force between tool and workpiece. The movements are generated by motors, transmission and guiding elements. These internal components cause heat losses due to friction and electrical processes. The manifold mechanisms of heat transfer, such as heat conduction in structural parts or convection between assemblies, lead the heat to the environment. This causes asymmetrical unsteady temperature fields and thermo-elastic deformations in the structural parts. The deformations lead to a displacement of the point of action via the kinematic coupling of the axle assemblies.

These processes are specific to the machine and the application. The reason for this lies in the wide structural variety of machine types and different operating conditions. The measures to improve the behavior must therefore also be analyzed and designed specifically for the machine and application.

\subsection{Approaches}

Analyses of this behavior are feasible both experimentally and simulatively, whereby a combined approach is appropriate. Experimental analyses are usually essential due to their high accuracy. Their capabilities are limited because of the restricted access to certain behavioral variables and the impact of constantly changing environmental conditions. Simulative investigations are not subject to these limitations. However, they can currently only partially replace experiments, because the effort to create and calculate the models is still considerable. The methods presented in the article are meant to help to reduce this effort.

Experimental analyses have so far mainly been used to determine the fundamental thermal and thermo-elastic character and the influence of components on the overall behavior of the machines. For this purpose, mainly measurements of temperatures and displacements of the outer structural components are performed [Gebhardt 2014, Ibaraki 2012]. However, the determination of causal quantities at inner components is hardly possible in this way.

One reason is the limited accessibility for measurements because of the encapsulated design of axle assemblies and the machine housing. Another reason is the high effort of experimental investigations. is the high time and metrological expenditure. Time expenditure results mainly from the large thermal time constants, which can reach several hours. The metrological efforts result from the installation of temperature and deformation measurement equipment as well as the setting up of defined thermal boundary conditions, which may require air-conditioned "thermal chambers" [Ihlenfeldt 2014].

Methods for the simulative analysis of the thermo-elastic behavior of machine tools are already developed. They allow accurate statements on the transient thermal behavior of typical machine tool structures with high structural resolution in a short time [Galant 2014]. Thus, an efficient analysis of the thermal behavior of machines with detailed structural components, internal heat sources and sinks as well as relative movements is possible in principle.

However, the methods have been developed with regard to correction applications. Correction models are created for one type of machine and can then be used for all produced exemplars. This can justify a higher effort in model creation. The computational speed of the correction models must allow at least real-time simulation. For thermal analyses, on the other hand, several design variants must be examined for their suitability. In order to keep the modeling effort acceptable, a modeling support tailored to machine tools is necessary. This support is currently not yet available. Furthermore, the design variants have to be evaluated with respect to relevant load regimes. These apply thermal loads corresponding to typical machining processes and can therefore last for long periods of time. A fast model execution is even more important here.

\subsection{Model types}

The thermo-elastic functional chain of machine tools and their system components can be simulated by network models [Steiert 2020]. The tools of digital block simulation (DBS) are preferably used for this purpose. In comparison to models based on Finite-Element Methods (FEM), the geometry of structural components is often coarsely reproduced. Using the network models, nonlinear behavior of heat dissipation sources and heat transfers of further system components can easily be integrated. Examples are models of electric drives [Dajaku 2006], gears and cooling channels [Steiert 2020]. Furthermore, the positiondependent heat transfer and heat input associated with the axis movements [Galant 2014] can be easily considered.

FE-models, on the other hand, offer advantages for the representation of structural components. With the available computer-aided engineering (CAE) tools, FE models can be generated largely automatically from CAD data. This is achieved by finely meshed geometries with very good approximation of the component shapes. This results in a very high number of degrees of freedom $\left(10^{5}\right.$ to $\left.10^{6}\right)$ and leads to very long simulation times. In order to shorten simulation times, the geometric details of structural components are therefore often greatly simplified. This reduces the number of mesh elements and thus the calculation time [Mayr 2012]. However, this procedure increases the effort for model creation and reduces the accuracy. A second method uses procedures for MOR. These allow a transformation of the models into systems with much lower degrees of freedom and thus lower computational load. This is achieved with very little loss of accuracy [Galant 2014]. 


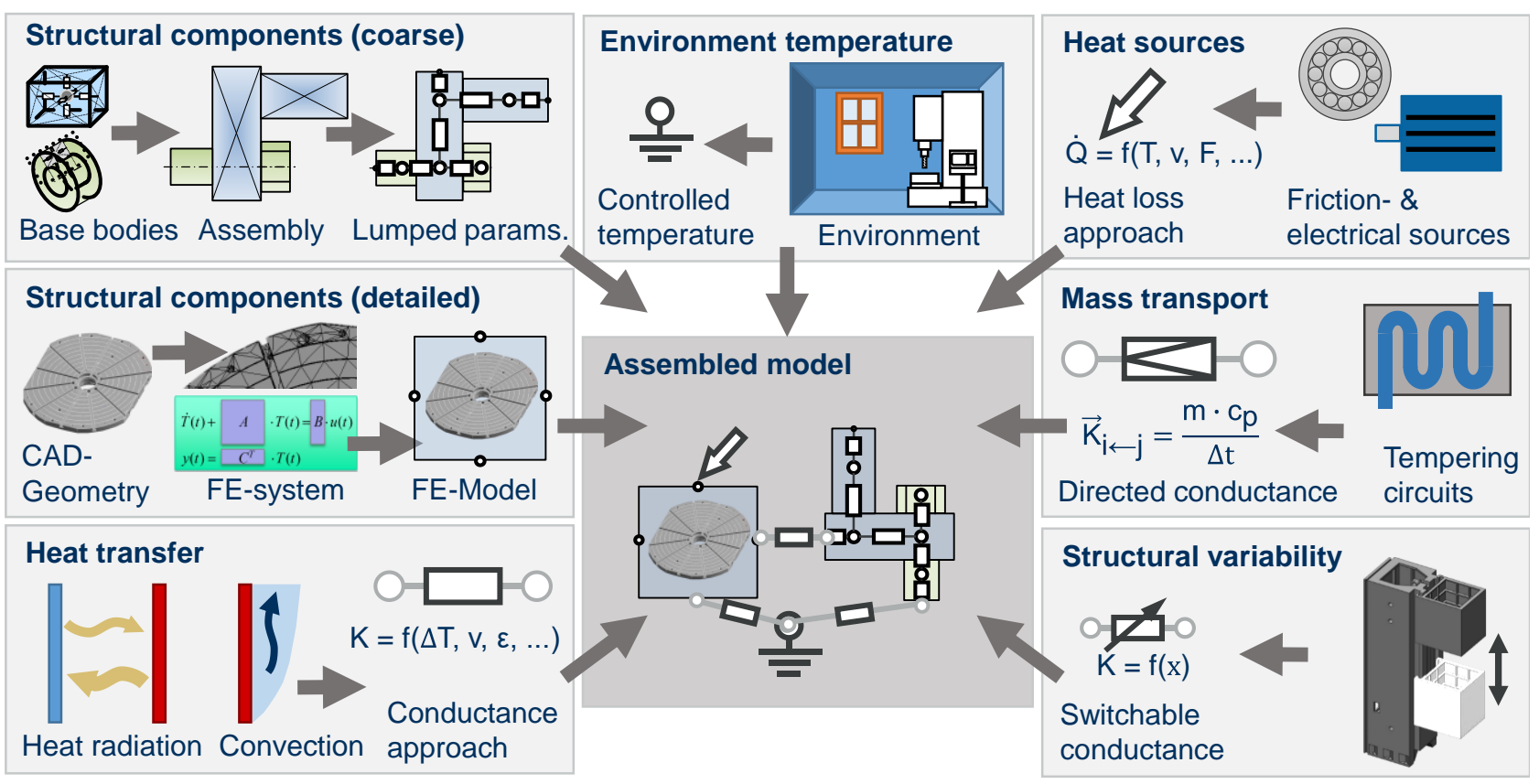

Fig. 2: Network model with machine specific model elements

\section{MODEL CONCEPT}

Currently available simulation tools are each specialized for one of the presented model types. Therefore, some system components cannot be integrated in a suitable model form in current analyses. They first have to be transformed or even simplified, which leads to high implementation effort and may result in significant lower accuracy. The modeling approach chosen here is based on a network model with model elements tailored to the behavior of machine tools (Fig. 2). The network approach allows on the one hand a combination of elements from different physical domains and elements of different model types on the other.

For the representation of structural components, two types of elements are provided, as suggested in [Schroeder 2018]. First, elements for lower geometric resolution. With these elements, components can be modelled using basic geometric bodies. These are then transferred into a lumped parameter model. The elements are suitable for rough analysis tasks in the conceptual and early design stages or for components with low overall impact. And second, FEbased elements. These allow high geometric resolution and use MOR methods to reduce the numerical effort. They can be used for analyses in the design stage where more detailed geometric designs are available. These elements are discussed in the subsequent sections.
The structural components exchange heat on the outer surfaces with other machine components or the environment. The heat transfer takes place by free and forced convection, by thermal radiation as well as over rolling contacts in bearings and guiding elements. Some of these processes behave in a very complex manner. For this reason, they are considered as independent model elements in the overall model, as shown in Fig. 2. This allows, for example, the use of different convection models starting from a simple constant through nonlinear empirical approaches up to fluid simulation models.

The machine is thermally connected to the surroundings in the workshop. This includes the ambient air, the radiation environment and the fundament. The temperature of these objects is defined as an external boundary condition. This is done time-dependently to take into account, for example, day-night fluctuations of the hall temperature.

The internal thermal load occurs as a result of heat losses. They are caused by friction in bearings and guideways or by electrical effects in drives. On the model side, they are mainly represented by empirical model approaches.

Model elements are needed for heat transport by mass transport, which is present e.g., in cooling channels. They are implemented as one directional thermal conductances in the lumped parameter models.

The last element considers the movement of the axle assemblies. This changes the location of the heat transfer between the surfaces which are moving relative to each

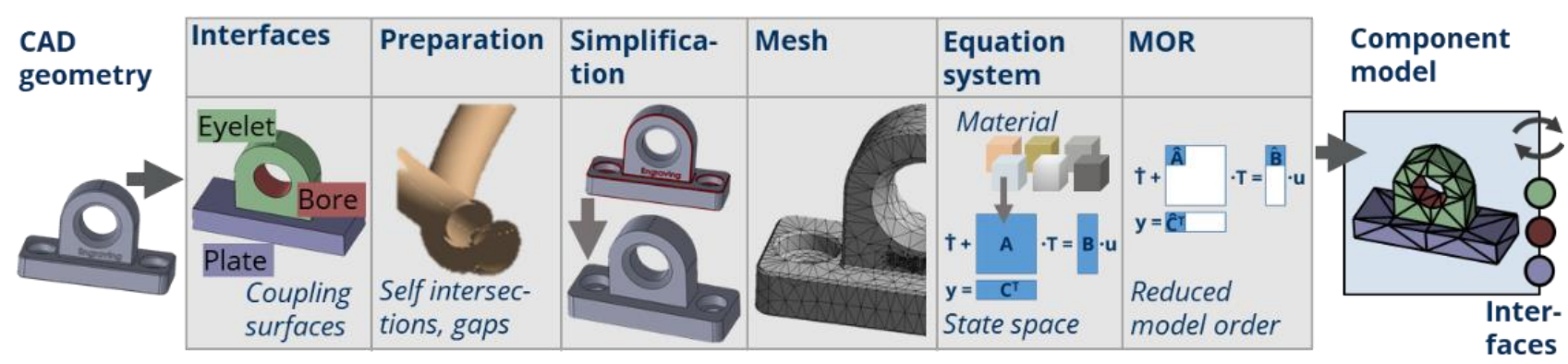

Fig. 3: Workflow for creation of FEM component models

MM Science Journal | 2021 | Special Issue on ICTIMT2021 
other and is taken into account using position-dependent heat transfers.
Coupling surfaces are partial surfaces of a component. The coupling surfaces in turn consist of several discrete surfaces that are combined in surface groups. They each have common heat exchange mechanisms and a common coupling partner. These are, for example, all surfaces that are connected by convective heat transfer to the ambient air. The selection of the discrete surfaces is a manual process, which is supported by selection assistance in CAD tools.

\section{FEM COMPONENT MODEL FOR STRUCTURAL PARTS}

The elements for geometrically high-resolution representation of the structural components are encapsulated building blocks. They describe the heat storage and conduction processes of solid bodies of the machine. The models are constructed with the help of FEM. From the point of view of thermal analysis, the following requirements apply to the creation and simulation of the models:

- Thermal machine models are very extensive. Many thermally relevant components have to be modelled. Therefore, a fast and thus automated generation of the FE models is necessary.

- In thermal analyses, several design variants are usually compared. This is done with regard to long-time loading regimes. Very fast computational models are required for this.

- For an easy applicability the models should have no excessive memory requirements.

\subsection{Sub-steps for model creation}

Figure 3 shows the workflow for model creation. It is derived from a classical FEM workflow and tailored to the aspects of the thermal analysis. Starting point is the CAD geometry of the components.

The thermal coupling with other simulation elements in the overall model (Fig. 2) is done via coupling surfaces (Fig. 3).
Before meshing the geometry for the geometric discretization process of the FEM, a preparation of the geometry data is often necessary. The reason are defects in the geometry description such as self-overlaps or gaps. The defects arise through conversion of data formats. The conversion is necessary because of the tool-specific geometry formats. An important step is therefore the correction of the geometry data. This can already be done automatically in some cases. However, time-consuming manual interventions are often necessary.

Furthermore, a geometry simplification is necessary, because the required computing resources of the models, by which computing load and memory requirements are understood, are considerable. This is not related to resources for the transient temperature calculation, because this is done with small reduced systems. Rather it is related to the generation of the FE-systems, the reduction of the degrees of freedom (DOF), the back projection of the calculation results to the original full system and the subsequent evaluation of the results in the time domain. The effort for all these operations depends on the DOF of the system. The number increases strongly with the reproduction of small geometry details. However, these have very little influence on the thermal behavior. Therefore, it is useful to simplify them.

Based on the refined geometry the differential equation system for the calculation of the thermal processes is created. For this purpose, the geometry for the FEelements is divided and the linear dynamic equation system
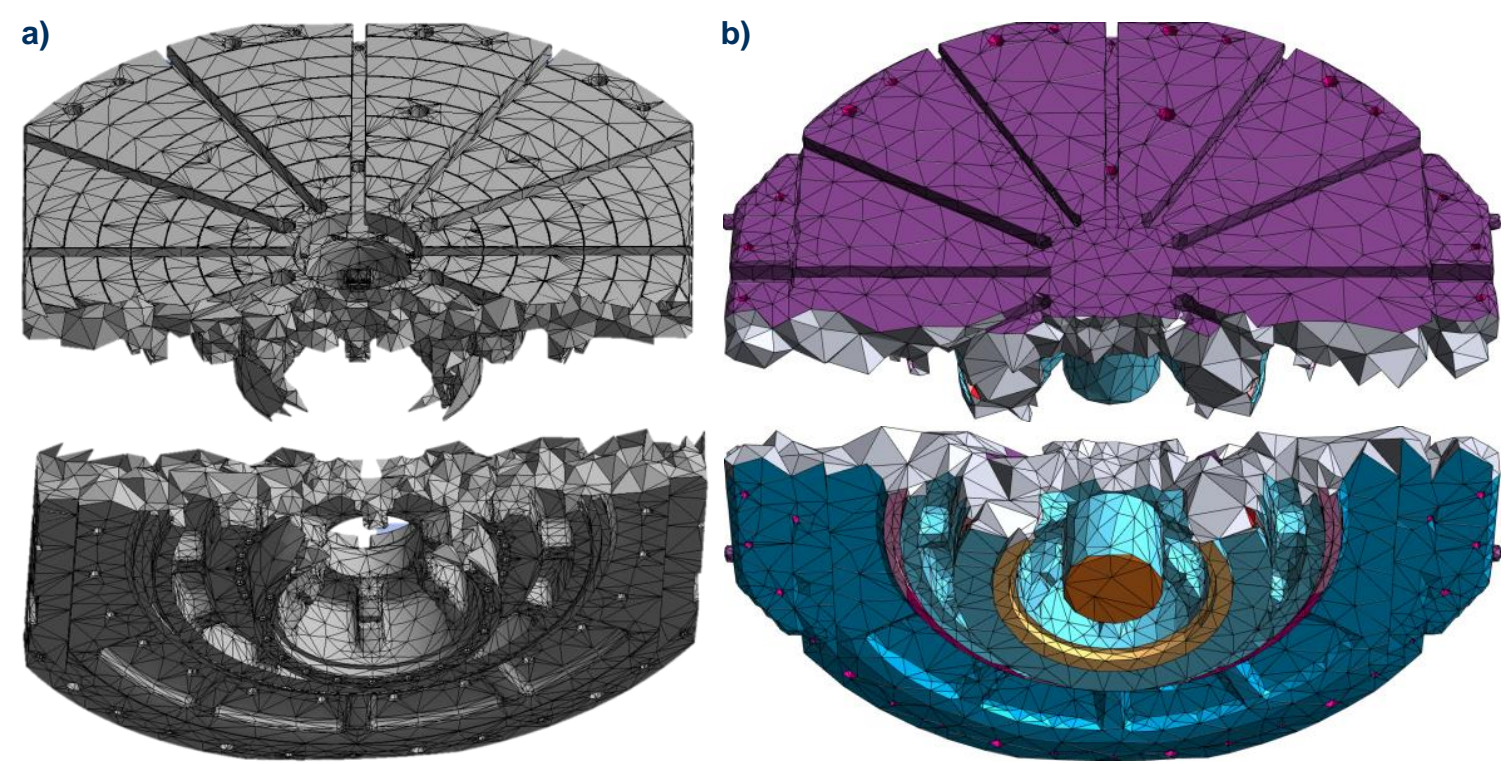

Fig. 4: Table plate of a machine tool; meshed by: a) ANSYS Mechanical, b) fTetWild 
is built. Finally, the system of equations is transformed into a fast-calculating system using mathematical MOR methods.

\subsection{Geometry simplification}

The number of nodes of the FE-mesh determines the DOF of a thermal equation system. For example, the mesh of the table component in Fig. 4 a) has a number of nodes $(n)$ of approximately 150000 . The memory requirement of the temperature field with floating point numbers of 8 bytes is 1.2 megabyte. The total memory requirement of the FEsystem is a multiple of this. The algorithmic computational complexity for the creation of the equation systems is up to $\mathrm{O}\left(\mathrm{n}^{3}\right)$. Summed up over all components of the machines, a high demand of computing resources results.

The challenge during geometry simplification is to achieve a low loss of accuracy while significantly reducing the required amount of computing resources. The geometry simplification can be divided into two steps, as Fig. 5 shows. In the first stage, only small geometry details such as engravings, chamfers or smaller holes are removed. In the second stage, smaller shape-determining geometry elements are eliminated. Tab. 1 compares the two steps for the part in Fig. 5 in terms of required computing resources and loss of accuracy. The evaluation of the computing resources is based on the number of nodes after meshing the component with tetrahedron elements. The evaluation of the loss of accuracy is done by comparing the time constants of the components. The time constants represent the temporal behavior during heat exchange with the environment. The time constants consider both heat storage and heat exchange processes. These in turn depend on the geometrical quantities volume and surface area.

The first stage shows an $86 \%$ reduction of computing resources with a loss of accuracy of $3.6 \%$. This loss of accuracy can be considered as very small with respect to the typical uncertainties of further sub models needed for a machine [Kauschinger 2014]. The second stage shows a considerable doubling of the model deviations with a small change of the required computing resources. This shows that the meshing of small geometric contours requires a disproportionate high number of tetrahedral elements. For a slightly refined geometry of stage 1 , the geometries are approximated with a few larger elements. This is associated with a small loss of accuracy. A further simplification of the geometry is not reasonable because of the increasing loss of accuracy and the small memory reduction.

\subsection{Robust meshing tool for automation}

Up to now, various tools with tailored algorithms have been used for the subtask of preparing and simplifying the geometry and the subtask of meshing. Manual intervention is required for the preparation and simplification of the geometry. With the use of the new meshing method according to [Hu 2020] these interventions can be avoided.

The method is characterized by a particularly high robustness against geometry errors, it generates a highquality mesh structure that enables high computational accuracy and it works fast. The computing time is comparable to the less robust tetrahedralization algorithms based on the Delaunay approach. Input of the algorithm is a triangle-based surface description. Parameters to control the processing are:

- $\quad$ a tolerance for the possible deviation of the surface of the target mesh (envelope around the input geometry),
- $\quad$ the ideal size of the edge lengths of the tetrahedra to be generated and

- the filter energy, which indicates the effort that is required to optimize the tetrahedral quality.

The method generates the mesh by an iterative improvement of the source mesh with alternating triangle insertion and mesh optimization [Hu 2020]

The method was tested for its applicability for the meshing of machine parts. The free open source tool fTetWild was used, in which the method is implemented. The table top of a machine tool was selected as the research object. It represents a typical machine geometry with fine structural details. The aim was to simplify the geometry and to achieve a high-quality mesh with a low number of elements. As parameters the tolerance of $2 \mathrm{~mm}$, the edge length of 10 $\mathrm{cm}$ and the filter energy of 20 were used. The result is shown in Fig. 4. Despite significant errors in the surface geometry of the table, automatic meshing was performed successfully. Chamfers and other smaller geometry elements were removed. As far as the geometry made it possible, large elements are inserted which have a good shape with respect to the calculation accuracy. The resulting number of elements is about 21000 , which is significantly reduced compared to the 150000 elements of the original geometry meshed with ANSYS Mechanical. The deviation of the volume is $0.4 \%$ and the deviation of the surface is $4.5 \%$. The meshing time was acceptable with 1 min.

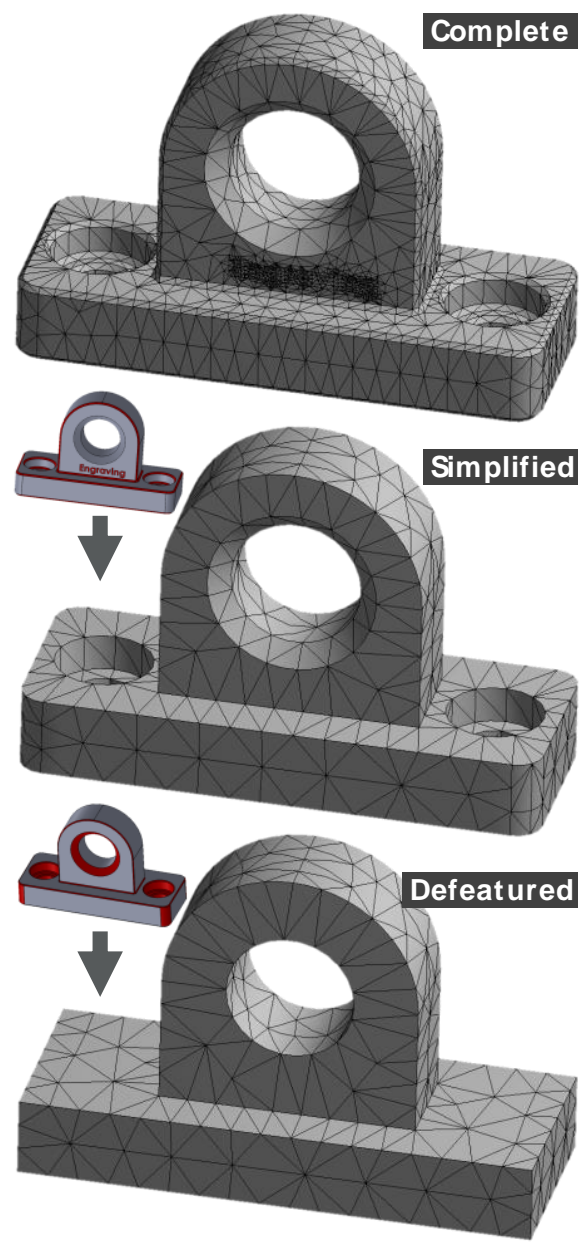

Fig 5: Two stage geometry simplification 
The handling of the tool is user friendly. It covers the problem-specific requirements with only three parameters. The degree of refinement can be controlled with the tolerance. The ideal edge length influences the discretization grade and the filter energy determines the computing effort to optimize the accuracy relevant quality of the mesh.

\subsection{Creation of equation systems}

Based on the refined geometry, the thermal equation system is discretized by means of FEM in the classical form:

$$
\mathrm{M} \cdot \dot{\boldsymbol{\tau}}+\mathrm{K} \cdot \boldsymbol{\tau}=\dot{\boldsymbol{q}}=\mathrm{D} \cdot \boldsymbol{u}
$$

$\mathrm{M}$ and $\mathrm{K}$ are thermal capacity and conductance matrices, $\boldsymbol{T}$ and $\dot{\boldsymbol{q}}$ are time-dependent temperature and load vectors. The capacitance and conductance matrices represent the geometry- and material-dependent storage and conductivity of heat, the load vectors describe the input or output heat. The load vectors are composed of the matrix $\mathrm{D}$, which represents the location of the heat input, and the vector $\boldsymbol{u}$, which contains the amount of heat. Loads are considered via Neumann (N) and Robin (R) boundary conditions. Neumann boundary conditions are to be understood as heat flows. They are used for the input of heat losses in the machines. Robin boundary conditions describe heat transfer to the environment (E) of the component, like convection at surfaces. As equation (2) shows, the heat transfer coefficients $(h)$ of the Robin boundary conditions are integrated into the conductance matrix, allowing the specification of external loads independent of the temperature of the solid. For this purpose, the conductance matrix $\mathrm{K}_{R}$ is required. It represents the surface geometry where the heat transfer takes place. The heat conduction of the solid itself is considered with $\mathrm{K}_{\mathrm{B}}$. The vector of the heat load is composed of the heat flows $\left(\dot{\boldsymbol{q}}_{\mathrm{N}}\right)$ for the Neumann boundary conditions and the product of heat transfer coefficients and the temperature of the coupled environment for the Robin boundary conditions.

$\mathrm{M} \cdot \boldsymbol{\tau}+\underbrace{\left(\mathrm{K}_{\mathrm{B}}+\mathrm{K}_{\mathrm{R}} \cdot \mathbf{h}\right)}_{\mathrm{K}} \cdot \boldsymbol{\tau}=\underbrace{\mathrm{D}_{\mathrm{N}} \mathrm{D}_{\mathrm{R}} \cdot \dot{\boldsymbol{q}}_{\mathrm{N}}}_{\mathrm{D} \cdot \boldsymbol{u}}$

In order to be able to integrate the system into an overall simulation, it is transferred into a state space representation with input and output signals (3). Input variables $u$ are the load vectors and output variables y are temperatures. The control matrix $B$ expresses the FE-nodes where the loads are applied. The measuring matrix $C$ defines whether the output signals represent temperatures of selected FEnodes or mean temperatures over a node area.

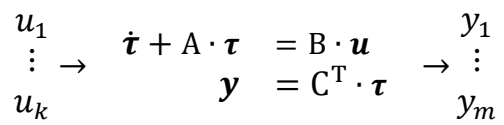

In order to calculate the system in a time-efficient way, it is transferred to a fast-computing system using MOR methods. A projection-based method is used. This projects the original system into an appropriate subspace. Thereby a system with the same structure is generated, but with a much smaller degree of freedom. The transient temperature field calculation is performed computationally efficient in this system. Afterwards the temperature field can be transformed back into the original system. The deviations regarding the calculations with the original model are comparatively small.

In the approach taken here the system is expressed as a combination of linear sub models (4). One subsystem per coupling surface $(k)$ is built, where Neumann or Robin boundary conditions can be used to define inputs. For each input $a$ vector $b$ is taken out of the columns of $B$ [Brecher 2019].

$$
\sum\left\{\begin{array}{cc}
\dot{\boldsymbol{\tau}}_{1}+\mathrm{A} \cdot \boldsymbol{\tau}_{1}=\mathbf{b}_{1} \cdot \boldsymbol{u}_{1} ; & \boldsymbol{y}_{1}=\mathrm{C}^{\mathrm{T}} \cdot \boldsymbol{\tau}_{1} \\
\vdots & \vdots \\
\dot{\boldsymbol{\tau}}_{\mathrm{k}}+\mathrm{A} \cdot \boldsymbol{\tau}_{\mathrm{k}}=\mathbf{b}_{\mathrm{k}} \cdot \boldsymbol{u}_{\mathrm{k}} ; & \boldsymbol{y}_{\mathrm{k}}=\mathrm{C}^{\mathrm{T}} \cdot \boldsymbol{\tau}_{\mathrm{k}} \\
\boldsymbol{y}=\boldsymbol{y}_{1}+\cdots+\boldsymbol{y}_{\mathrm{k}} &
\end{array}\right.
$$

Within the context of MOR, a transformation matrix $\mathrm{V}$ is generated for each of these subsystems using the classical Arnoldi algorithm. With these transformation matrices the subsystems are projected into a Krylov subspace with a small dimension $n(5)$. The temperature field calculation is now performed with these subsystems. The approximated solution of the full system is obtained by superposition of the re-transformed solutions of all subsystems (6) [Galant 2014].

$$
\begin{array}{ccc}
\mathrm{A}, \mathbf{b}_{1} & \stackrel{\text { Arnoldi }}{\longrightarrow} \mathrm{V}_{1}^{n} & \rightarrow \widehat{\mathrm{A}}_{1}, \hat{\mathbf{b}}_{1} \widehat{\mathrm{C}}_{1} \\
\vdots & \vdots & \vdots \\
\mathrm{A}, \mathbf{b}_{\mathrm{k}} & \stackrel{\text { Arnoldi }}{\longrightarrow} \mathrm{V}_{k}^{n} & \rightarrow \widehat{\mathrm{A}}_{\mathrm{k}}, \hat{\mathbf{b}}_{\mathrm{k}} \widehat{\mathrm{C}}_{\mathrm{k}} \\
\boldsymbol{\tau}=\mathrm{V}_{1}^{n} \cdot \hat{\boldsymbol{\tau}}_{1}+\cdots+\mathrm{V}_{k}^{n} \cdot \widehat{\boldsymbol{\tau}}_{\mathrm{k}}
\end{array}
$$

\subsection{Modularization through open interfaces}

To ensure that the workflow for creating structural component models can be transferred to the diverse tool chains of potential users, it is designed in a modular way. The individual work steps are carried out with tailored tools. The tools are coupled by common interfaces. This makes the tools interchangeable. In order to achieve the highest possible acceptance of the interfaces, standardized or open and documented interfaces are used.

A description of the surface geometry is required as input variable of the first step of the workflow. It should be possible to accept assemblies of rigidly connected parts. These assemblies can be treated as merged parts during the thermal simulation. The merging is possible because the gaps at the part connections usually have a low overall thermal effect. In the next step the coupling surfaces must be selected on the surface of the assemblies. The effort depends on the form of geometry representation. With the BREP (Boundary Representation) format, body surfaces are generated via interconnected surfaces. The surfaces are stored as a mathematical description and can adopt a wide variety of shapes and thus also represent curvatures

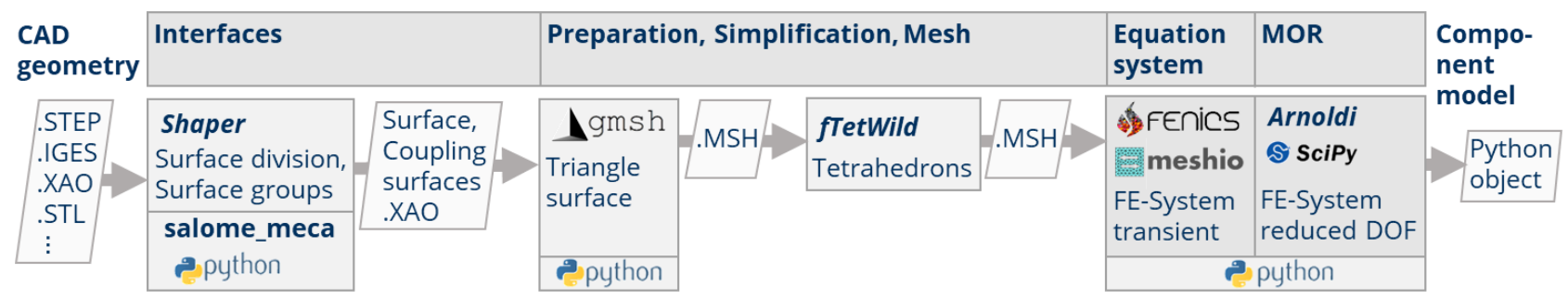

Fig.6: Modularized tool chain 
well. Using formats with polygons, this is not possible. Curved surfaces usually need many polygons to approximate their shape. Since the surfaces with BREP format are usually represented with fewer sub-surfaces, fewer sub-surfaces must be selected for the coupling surfaces. This format is therefore to be preferred. A BREP format that meets the requirements is the standardized STEP format. It is supported by most CAD and FEM programs. IGES is another standardized BREP format. It is not so widely supported and it is generated more often with surface defects.

As input information for meshing, the surface geometry of the assembly must be provided in the form of the coupling surfaces. In many FE and CAD programs there is the possibility to define surface groups. In principle it is also possible to transfer such information with the often-used STEP or IGES formats. So far this is hardly supported. In many tools therefore mostly own data formats are used. The open-source FE program Salome Meca for example uses the open and documented XAO format. In commercial programs the exchange formats are usually not documented.

Alternatively, the coupling surfaces can be exchanged in mesh files. Mesh files are used in the FEM to describe the geometrically discretized surfaces and volumes. It is common to define boundary conditions on surfaces and to store this information in the files. Thus, mesh files are suitable as an interface for transferring both, the part geometry and the coupling surfaces. Common open and documented data formats are CGNS, MED, and MSH. The disadvantage is that none of these formats is widely supported. However, there are converters like Meshio that can translate these formats.

Fig. 6 shows the example implementation of the tool chain for the generation of structural component models. Open and documented tools and interfaces have been used throughout, which allows an easy exchangeability of the tools. The tools have easy to use and documented Python interfaces. The tools are embedded in the higher-level modeling tool through these interfaces. This made it possible to automate the model creation process almost entirely.

\subsection{Verification of the MOR-FEM simulation}

To verify the workflow, an exemplary simulation of the cooling of a steel plate was performed (Fig.7). This represents the cooling of a wall of a machine tool frame component heated by internal heat sources. The heat stored in the plate is released over the surfaces. Except for one of the small end faces. During cooling, the plate approaches the ambient temperature $T_{\text {env }}$.
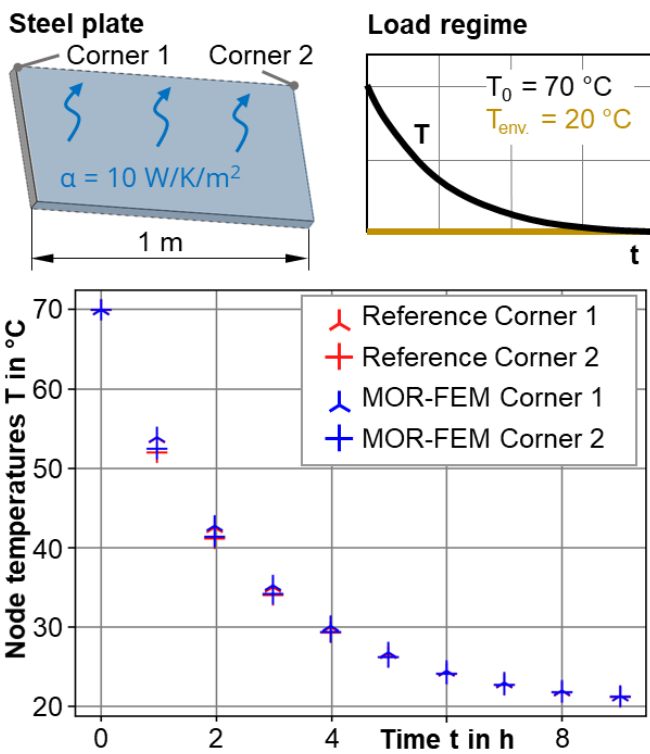

Fig.7: Verification model and simulation results

The order-reduced model is generated from a highresolution base model and has a DOF of 20 . The model accuracy is verified using two simulated node temperatures at the plate corners. The temperatures are determined by a re-transformation of the virtual temperatures calculated with the reduced model.

The comparison of the temperature with a temporally and geometrically highly resolved reference model with a DOF of approx. 62000 shows only small deviations. The maximum deviation appears shortly after the temperature step with an amount of $0.44 \mathrm{~K}$. The computing time of the reduced model is more than 100 times shorter than that of the reference model.

\section{SUMMARY AND OUTLOOK}

The article presents methods to support the simulationbased thermal analysis of machine tools. At first, requirements and state-of-the-art modeling techniques are presented. A modeling approach is derived, in which thermal models are composed of domain-specific elements. This reduces the abstraction effort during model generation compared to generically oriented model elements

Subsequently, a model element for a geometrically detailed representation of structural components is presented. By using a robust meshing tool, the preparation and refinement of the geometry is automated. The workflow for model generation is modularly structured. The tools involved are connected by standardized open and documented interfaces. In this way, the presented workflow can be integrated in existing tool-chains of machine manufacturers. And finally, the methods for MOR reduce the calculation effort and thus significantly shortens the usually long calculation times.

The aim of further work is to extend the numerical tool for all essential thermal behavior components. To accomplish this, the library of model elements for the network model (Fig. 2) will be completed. One of the main challenges is the efficient simulation of the behavioral components coupled within an overall model. This includes the calculation of:

- lumped parameter models for coarsely modeled structural components,

- MOR-FE-models for detailed geometric reproduction of structural components, 
- numerous nonlinear models for heat transfer and heat losses and

- forced heat flows for cooling circuits.

Finally, the tool will be tested by analyzing compensation measures for a current 5-axis machine tool. Among other things, cooling concepts of a table with rotation and tilt axes will be evaluated with respect to their cooling effectiveness.

\section{ACKNOWLEDGEMENTS}

The German Science Foundation (DFG) funded this research within the CRC 96 "Thermo-energetic design of machine tools" project T05.

\section{REFERENCES}

[Brecher 2019] Brecher, C., Ihlenfeldt, S., Neus, S., Galant, A. and Steinert, A. Thermal condition monitoring of a motorized milling spindle. Production Engineering Research and Development, 2019

[Donmez 2007] Donmez, M.A., Hahn, M.H., Soons, J.A., 2007. A Novel Cooling System to Reduce ThermallyInduced Errors of Machine Tools. CIRP Annals 56, 521524.

[Dajaku 2006] Dajaku G, Gerling D. An improved lumped parameter thermal model for electrical machines. In 17th International conference on electrical machines (ICEM2006). 2006.

[Galant 2014] Galant, A., Großmann, K., and Muehl, A. Thermo-elastic simulation of entire machine tool. Thermoenergetic Design of Machine Tools, Berlin u.a., 2014, pp. 69-84.

[Gebhardt 2014] Gebhardt M., Knapp W, Schneeberger A, Weikert S, Wegener K. Thermally Caused Location Errors of Rotary Axes of 5-Axis Machine Tools. IJAT. 2014 8:51122.

[Hu 2020] Hu, Y., Schneider, T., Wang, B., Zorin, D. and Panozzo, D. Fast Tetrahedral Meshing in the Wild. arXiv:1908.03581 [cs], Jan. 2020

[lbaraki 2012] Ibaraki S, Hong C. Thermal test for error maps of rotary axes by R-test. Key Engineering Materials. 2012; 523-524:809-14
[Ihlenfeldt 2014] Ihlenfeldt S, Richter C, Schaedlich K; Naumann C. Erfassung und Modellierung der thermischen Wechselwirkungen von Umgebung und Maschine. ZWF Zeitschrift fuer wirtschaftlichen Fabrikbetrieb. 2014;109(78):526-29

[Kauschinger 2014] Kauschinger, B. and Schroeder, S. Methods to Design the Adjustment of Parameters for Thermal Machine-Tool Models. Proceedings of the WGP Congress 2014, Erlangen, 2014, vol. 1018, pp. 403-410.

[Kauschinger 2016] Kauschinger, B., Schroeder, S.: Uncertainties in heat loss models of rolling bearings of machine tools. Procedia CIRP 7th HPC 2016, CIRP Conference on High Performance Cutting Chemnitz, 2016 [Mayr 2012] Mayr J, Wegener K, Uhlmann E, Brecher C, Knapp W, Hartig F, et al. Thermal issues in machine tools. CIRP Annals - Manufacturing Technology. 2012;61(2):77191.

[Mayr 2018] Mayr, J., Blaser, P., Ryser, A., HernandezBecerro, P., 2018. An adaptive self-learning compensation approach for thermal errors on 5-axis machine tools handling an arbitrary set of sample rates. CIRP Annals 67, 551554.

[Mori 2009] Mori, M., Mizuguchi, H., Fujishima, M., Ido, Y., Mingkai, N., Konishi, K., 2009. Design optimization and development of CNC lathe headstock to minimize thermal deformation. CIRP Annals 58, 331-334.

[Putz 2018] Putz M, Richter C, Regel J, Braunig M. Industrial Relevance and Causes of Thermal Issues in Machine Tools. Conference on Thermal Issues in Machine Tools Proceedings 2018, Wissenschaftliche Scripten, Auerbach /Vogtl, 127-139.

[Schroeder 2018] Schroeder, S., Galant, A., Beitelschmidt, $M$. and Kauschinger, B. Efficient modelling and computation of structure-variable thermal behaviour of machine tools. Conference on Thermal Issues in Machine Tools, Auerbach Nogtl, 23.03 2018, pp. 13-22.

[Steiert 2020] Steiert, C., Weber, J., Galant, A., Glanzel, J., and Weber, J. Fluid-Thermal Co-Simulation for a Machine Tool Frame. presented at Internationales Fluidtechnisches Kolloquium IFK2020, Dresden, Mar. 202 